\title{
Migration of Indwelling Central Venous Catheter and Fatal Hydrothorax
}

\author{
Shagufta Jabeen ${ }^{1}$ Ghulam Murtaza ${ }^{1}$ Muhammad Zubair Hanif ${ }^{2,3}$ Antonino Morabito ${ }^{1}$ Basem Khalil ${ }^{1}$
}

1 Department of Paediatric Surgery, Royal Manchester Children's Hospital, Manchester, United Kingdom

2 Department of Cardiovascular Surgery, Shandong Provincial Hospital, Shandong University, Jinan, Shandong, China

${ }^{3}$ Department of Cardiac Surgery, Linyi People's Hospital, Shandong University, Linyi, Shandong, China

Eur J Pediatr Surg Rep 2014;2:32-34.
Address for correspondence Shagufta Jabeen, MRCS, Department of Paediatric Surgery, Royal Manchester Children's Hospital, Oxford Road, Manchester M13 9WL, United Kingdom (e-mail: sheenjeem1@gmail.com).
Abstract
Keywords
- central venous catheter insertion
- complications
- migration
- perforation
- hydrothorax

Central venous catheter complications can be related to insertion, indwelling, or extraction. Most of the times, immediate complications are anticipated and managed; whereas, delayed complications can go unnoticed. In the case discussed here, migration and dislodgement of catheter tip resulted in delayed hydrothorax and sudden death of a 9-month-old female infant.

\section{Introduction}

Central venous catheter (CVC) insertion is one of the most commonly performed procedures in pediatric surgical theaters. Central line is required in patients on repeated intermittent drug therapy, regular transfusions, total parenteral nutrition (TPN) and chemotherapy, etc. Long-term indwelling central lines remain a constant source of delayed complications irrespective of continued surveillance. These complications include infection, thrombosis, fracture and embolization, catheter migration, venous erosion and hydrothorax. Catheter material, postural movements, chemical irritation, and female gender are some of the important risk factors. There is a need for continuous monitoring and research to avoid possible risk factors so as to minimize the morbidity and mortality.

\section{Case Report}

A white British female infant was born at 36 weeks and 6 days gestation with antenatal diagnosis of gastroschisis. She was taken to theater the same day for silo closure of gastroschisis and open insertion of 2.7 French (Fr) CVC in right internal jugular vein (IJV) for TPN administration. This catheter was replaced with a long line, sited percutaneously in right long saphenous vein because of catheter blockage at approximately 2 months of age. She developed necrotizing enterocolitis soon after; henceforth, a new 4.2-Fr Hickman line was inserted in left external jugular vein via open technique, and the position of tip was confirmed on intraoperative radiograph (-Fig. 1). She underwent a couple of operations during her 7.5-month stay at hospital and was discharged on home TPN. She was regularly reviewed by our dietician and specialist nurses at home with satisfactory reports. Six weeks postdischarge, she passed away without any obvious reasons.

Postmortem examination showed considerably sized perforation in the wall of left subclavian vein (SCV) with marked perifocal edema of surrounding soft tissues while tip of Hickman line was within left axillary vein with minor injury to its wall. Left lung was compressed to half of its volume and was surrounded by approximately $100 \mathrm{~mL}$ of turbid milky fluid. Autopsy report suggested that death was caused by compression of left lung because of accidental perforation of left SCV and subsequent accumulation of the fluid in left pleural space. received

April 9, 2013

accepted after revision

April 16, 2013

published online

May 9, 2013

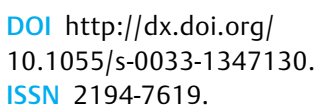

DOI http://dx.doi.org/

10.1055/s-0033-1347130. ISSN 2194-7619.

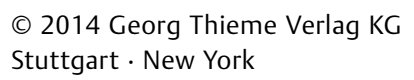

License terms

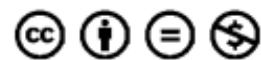




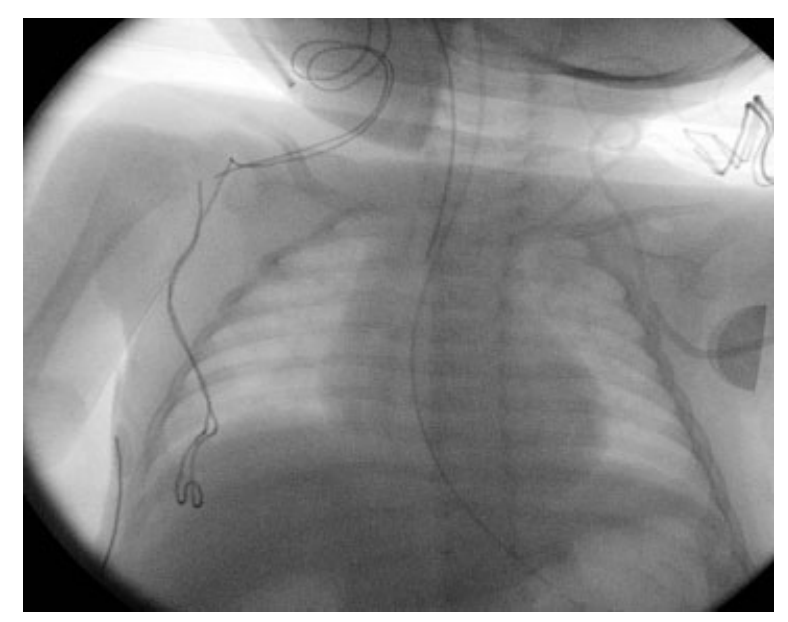

Fig. 1 Intraoperative $X$-ray image showing the tip of central venous catheter in left brachiocephalic vein.

Our case is exceptional where CVC was placed in external jugular vein with its tip at the junction of superior vena cava and right atrium that later on migrated to the left SCV, causing a huge perforation with lodgment of the tip in the lumen of left axillary vein. Resultant hydrothorax proved fatal irrespective of continuous care and follow-up.

\section{Discussion}

CVC insertion is a common procedure performed on a wide range of critically ill and chronic patients. It gives us an opportunity to assess their hemodynamic status, to infuse TPN, chemotherapy, rapid volume expanders, and helps in hemodialysis. Internal jugular, external jugular, subclavian, and femoral veins are most commonly used veins for catheter placement. The procedure is performed through anatomical landmark technique, open surgical procedure, and ultrasound-assisted insertion. The catheters can be tunneled, like Hickman line and Portacath, or nontunneled, for example temporary catheters and peripherally inserted central catheters (PICC). Temporary catheters are usually inserted in emergency situations and are kept for about a week. A PICC is used in patients requiring weeks or months of access. Hickman lines are meant for longer durations and have an advantage of having fewer chances of dislodgment and infection.

The patients with CVC may suffer from certain complications that can be divided into immediate and delayed complications. The immediate complications occur either during catheter insertion or just afterward and can be divided into guidewire-related and catheter-related injuries. Guidewirerelated complications include arrhythmias, looping, kinking, and knot formation in the vessel lumen and even beyond. ${ }^{1}$ Entanglement of guidewire into the existing intravascular apparatus is another guidewire-related injury. Arterial puncture, pseudoaneurysms, arteriovenous fistulas, ${ }^{2}$ vascular and cardiac perforation, hematoma, hydrothorax, cardiac tamponade, tension pneumothorax, and air embolism are usually catheter insertion complications. The delayed complications are related to indwelling and extraction practices of the catheter and include infection, thrombosis, catheter malfunction, catheter migration, vascular erosion, retained intravascular catheter fragments, ${ }^{3}$ and formation of calcified cast in a long-term indwelling CVC. ${ }^{4}$

The position of catheter tip may change with respiration, blood stream flow dynamics, ${ }^{5}$ postural rotation, and neck movements. This mechanical friction may lead to vascular erosion and perforation. Chemical irritation of the vessel wall because of high osmolar solutions, like TPN, is another significant contributing factor. ${ }^{6}$

The selection of left-sided vessels for catheter placement is an independent risk factor because catheters are more likely to abut the right wall of superior vena cava at a sharp angle resulting in endothelial damage and subsequent vascular erosion and hydrothorax. ${ }^{7}$ Female gender may be another risk factor for erosion because of smaller size of the vessels. ${ }^{8}$ Earlier studies have implicated catheter material, size and their stiffness in vascular injuries, such as, polyethylene, as compared with their newer counterparts, such as, polyurethane. Nowadays, most catheters in practice are made up of polyurethane with probable decrease in incidence. Lack of expertise and number of needle passes also add to the rate of complications.

Literature review revealed that complication rate varies significantly ranging from 5 to $19 \%{ }^{9}$ Infection rate is 5.3 of 1,000 catheter days. Thrombosis rate is $1.9 \%$ for catheterization; whereas, it greatly increases to above 33\% for indwelling catheters. Superior vena cava obstruction is estimated to be 1 of 1,000 indwelling devices. Carotid artery puncture is reported to be 6 to $25 \%$, and up to $40 \%$ of these cases are associated with hematoma formation. Subclavian artery perforation occurs in approximately 0.1 to $1 \%$ cases. In cases of cardiac tamponade, mortality appears to be $90 \%{ }^{10}$ The incidence of brachial plexus punctures is approximately $1.7 \%$. Femoral catheterization is associated with more mechanical complications as compared with IJV or SCV catheterization. SCV is better off than IJV in elective and emergency procedures for mechanical complications; however, pneumothorax is more common in SCV, which is reported to be 1 to $1.5 \%{ }^{9}$

Hydrothorax is a delayed complication, which may present as shortness of breath, chest pain, hypotension, and decreased urine output. Chest X-Ray and ultrasound depict as pleural effusion, lung collapse, and widening of mediastinum; however, delayed hydrothorax may be less obvious clinically as compared with early insertion-related perforation where hemodynamic status of the patient is a well-anticipated indicator. The consequences of delayed hydrothorax could be fatal. ${ }^{11}$

Central venous catheterization is an excellent approach for venous access; however, it is associated with few delayed and dreadful complications. Right-sided vascular approach should be preferred to avoid some of the inherent friction because of sharp anatomical angle of superior vena cava for left-sided approach. There is an enhanced need of continuous research to develop more pliable and safer catheter materials and increased surveillance to detect these complications at an early stage to avoid rare and fatal complications. 
34 Migration of Indwelling CVC and Fatal Hydrothorax Jabeen et al.

\section{Conflict of Interest}

None

\section{References}

1 Khasawneh FA, Smalligan RD. Guidewire-Related Complications during Central Venous Catheter Placement: A Case Report and Review of the Literature. Case Reports in Critical Care, 2011, ID 287261. Available at: http:/www.hindawi.com/crim/cc/2011/ 287261/. Accessed February 2, 2013

2 Guilbert MC, Elkouri S, Bracco D, et al. Arterial trauma during central venous catheter insertion: Case series, review and proposed algorithm. J Vasc Surg 2008;48(4):918-925, discussion 925

3 Milbrandt K, Beaudry P, Anderson R, Jones S, Giacomantonio M, Sigalet D. A multi-institutional review of central venous line complications: retained intravascular fragments. J Pediatr Surg 2009;44(5):972-976

4 Hughes DB, Ullery BW, Spigland N. Formation of a calcified "cast" in a long-term indwelling central venous catheter: a case report. J Pediatr Surg 2006;41(11):1927-1929
5 Domino KB, Bowdle TA, Posner KL, Spitellie PH, Lee LA, Cheney FW. Injuries and liability related to central vascular catheters: a closed claims analysis. Anesthesiology 2004;100(6):1411-1418

6 Girgin NK, Arici S, Turker G, Otlar B, Hotaman L, Kutlay O. Delayed pneumothorax and contralateral hydrothorax induced by a left subclavian central venous catheter: a case report. Clinics (Sao Paulo) 2010;65(5):562-565

7 Kunizawa A, Fujioka M, Mink S, Keller E. Central venous catheterinduced delayed hydrothorax via progressive erosion of central venous wall. Minerva Anestesiol 2010;76(10):868-871

8 Walshe C, Phelan D, Bourke J, Buggy D. Vascular erosion by central venous catheters used for total parenteral nutrition. Intensive Care Med 2007;33(3):534-537

9 Kusminsky RE. Complications of central venous catheterization. J Am Coll Surg 2007;204(4):681-696

10 Rajinikanth J, Stephen E, Agarwal S. Complication of central venous cannulation. Can J Surg 2008;51(5):E113-E114

11 Lefrant JY, Muller L, De La Coussaye JE, et al. Risk factors of failure and immediate complication of subclavian vein catheterization in critically ill patients. Intensive Care Med 2002;28(8): 1036-1041 